\title{
Evaluation of Model-Based Tracking and its Application in a Robotic Production Line
}

\author{
Fabian Scheer \\ Daimler Protics $\mathrm{GmbH}$ \\ Fabian.scheer@daimler.com \\ Marvin Ginader \\ Daimler AG \\ Marvin.ginader@daimler.com
}

\author{
Markus Neumann \\ Daimler Protics $\mathrm{GmbH}$ \\ Markus.n.neumann@daimler.com \\ Yavuz Oezkurt \\ Daimler AG \\ yavuz.oezkurt@daimler.com
}

\author{
Konrad Wirth \\ Daimler AG \\ Konrad.wirth@daimler.com \\ Stefan Mueller \\ University of Koblenz-Landau \\ stefanm@uni-koblenz.de
}

\begin{abstract}
The assessment of the accuracy and stability of model-based tracking approaches in the literature is a challenging task. Many of the presented methods lack a detailed evaluation or comparison to accurate ground truth data. Considering real world applications, it is hard to estimate the applicability of the respective method. In this paper we present methods to evaluate the accuracy and stability of model-based tracking approaches. Thereby, we focus on an industrial use and the robustness of the approach considering error sources like the influence of lighting over time, noise and the mechanical camera setup in general. The second part of this paper deals with the evaluation and application of model-based tracking in a robotic production line for gap measurements in the automotive industry. We present a method for synchronization with a conveyor setup and an operating robot. In this complex setup various error sources influence the output accuracy of the system. A further accuracy evaluation method is presented that uses the robot as a measurement device of the total error. The paper closes with the application of a model-based tracking system for robotic measurement tasks in a production line.
\end{abstract}

\section{Keywords}

evaluation, model-based tracking, evaluation methods, synchronization to conveyor, robotic production line

\section{INTRODUCTION}

While doing the research in the literature of modelbased tracking (MBT) approaches we found that most presented papers lack a detailed evaluation concerning the achieved accuracy in comparison to ground truth data. The influence of different camera resolutions, camera types or the influence of interferences like lighting, noise or mechanical setup is often poorly evaluated or different non comparable evaluation methods are used. With this in mind we present a tool set of methods in this paper to evaluate MBT techniques. For the research community and the industry this toolset can be of special interest to assess the accuracy, stability and feasibility of a MBT approach for their desired use case. Correlations between different error sources and their influence to the results become clearer and are easier to evaluate. To allow researchers the evaluation of their own MBT approach we plan to publish a CAD model of a test

Permission to make digital or hard copies of all or part of this work for personal or classroom use is granted without fee provided that copies are not made or distributed for profit or commercial advantage and that copies bear this notice and the full citation on the first page. To copy otherwise, or republish, to post on servers or to redistribute to lists, requires prior specific permission and/or a fee. specimen, camera intrinsic parameters, a video sequence of shifts of the specimen and the associated ground truth data recorded with a precise measurement device.

Automated industrial vision based solutions in cooperation with robots that work on a conveyor become more and more important in the context of future industrial manufacturing and are accompanied by several international initiatives like industry 4.0 , smart factories or the industrial internet consortium. In this regard MBT can play an important role but several challenges have to be mastered. Based on this motivation this paper presents a method to synchronize the vision based MBT with a conveyor and robots. The synchronization is evaluated in an application scenario of automatic measuring of gaps on body shells with a light weight robot.

\section{RELATED WORK}

Garon et al. [Gar18] gives a good overview over the current state of the art of frameworks and datasets for the evaluation of six degrees of freedom object trackers. Derived from the drawbacks of existing methods they propose a new method based on ground truth data captured with a Vicon motion capture system. They use complex error-prone transformation chains for their setup with a Microsoft Kinect. It is known that vision systems in combination with 


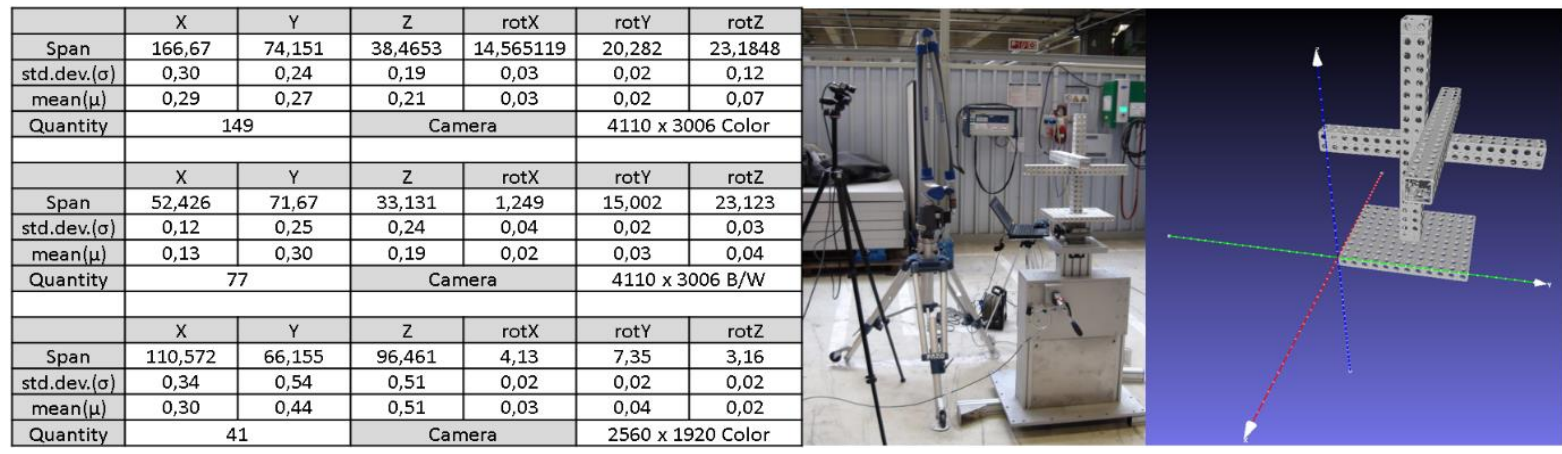

Figure 1: Results for Faro arm and test specimen. 3 different camera resolutions are used. Translations are in millimeters and rotations in degrees. Right: Experimental setup and scanned model of test object.

capturing systems like Vicon need calibration procedures that afflict the outcome results. Due to that, the results are mostly in the millimeter $(\mathrm{mm})$ range. Devices of the measurement technology achieve better results and are in the submillimeter range. An independent measurement solution regarding the sensor technology for the ground truth data would be more appropriate.

Several evaluation techniques use fiducials like [Hin11] or a checker board [Wu17]. [Hod17] also gives a good overview of the state of the art regarding datasets for the evaluation of 6D pose estimation for texture less objects. Unfortunately they also use fiducials and manually aligned poses to define ground truth data.

The existing methods try to combine a lot of test cases for an evaluation, like textured or untextured objects, robustness to occlusion or clutter, detection rates, training of artificial intelligence methods and so on. As already mentioned in [Gar18] most evaluation datasets and frameworks lack high precise ground truth data which is comparable to the results of professional measurement technology. Therefore we present methods that rely on ground truth data captured with an independent measurement technology. The setup is kept simple and designed in a way that the results can be compared directly.

For our evaluation we use the model-based tracking approach of [Wue07] that is based on [Com06] and [Vac04]. The method was further developed by the authors and has reached a high degree of maturity. This was important in our selection process since we are focused on an industrial use case. We only considered one MBT method because of the great expense for all evaluations presented in this paper. For initialization, [Wue07] rely on a real camera perspective that closely matches a given CAD perspective. They use a real-time image space line model generation method as detailed in [Nie03]. After the matching in the initial position a continuous tracking can be achieved. In Figure 2 the right image shows an augmentation of an image with the MBT.

\section{EXPERIMENTAL SETUP AND EVALUATION RESULTS}

Our evaluation consists of different methods to determine the accuracy of a MBT approach. We used precise measurement devices like a Faro arm [Far20] and a Faro laser tracker [Far20] to obtain ground truth data. Various test objects are used in the tests: a test specimen constructed with standard mechanical engineering elements of aluminum, car body shells and assembled coated cars. To keep things simple and avoid complex transformation chains we focused on the evaluation of a simple transform of the test object. Thereby, the pose of the test object was measured in an initial position. Then the object was moved and measured again. The transformation between the two poses is our evaluation criterion. The MBT delivers results in the coordinate system of the CAD object. The same CAD model of the test object was used in the calibration of the ground truth measurement system to the test object. With this setting, we avoid complex coordinate system calibrations. That makes the transformation of the ground truth and the MBT data directly comparable.

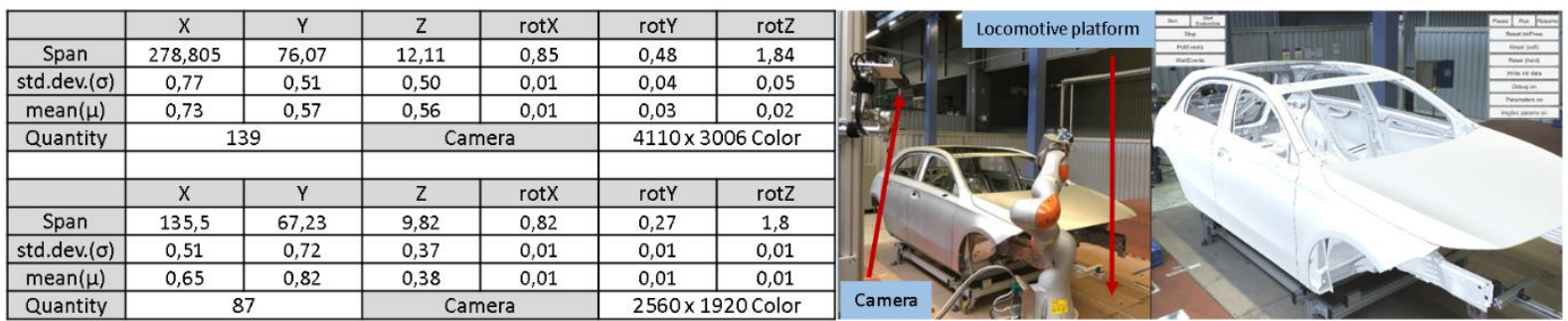

Figure 2: Evaluation results with Faro arm, Faro laser tracker and body shell. Two camera resolutions are tested. The measurements are in millimeter for the translation and in degrees for the rotation. 
We tested the MBT with a camera resolution of 4110 x 3006 (12 megapixels (MP)) and 2560 x 1920 (4 MP) pixels. A uEye UI-3000SE-C-HQ USB 3.1 camera with global shutter and $12 \mathrm{~mm}$ lens, as well as the same camera as black and white version was used. For the 2560x1920 pixel resolution a UI-3580CP-C-HQ Rev.2 USB 3.0 camera with a $6 \mathrm{~mm}$ lens was used.

\subsection{Faro arm with test specimen}

The first experiment was conducted with a test specimen assembled of robustly connected standard aluminum elements from the mechanical engineering sector. For ground truth measurements a Faro arm was used.

\subsubsection{Test setup}

To avoid errors related to the CAD modeling process, the test specimen was scanned with a high precision GOM 3D scanner [Gom20]. The scanned CAD model was used for the MBT and for the calibration of the faro arm to the test specimen. The extent of the specimen was about $300 \mathrm{~mm}$ on the longest side and the temperature during the measurements was in the range of 20 to 30 degrees Celsius. Therefore linear expansions caused by temperature fluctuations lie in the range of approximately $0.07 \mathrm{~mm}$ and could be neglected for the evaluation. As described above, the test specimen was measured in an initial position by the Faro arm and the MBT. Then the object was moved and measured again with both systems. Thus, the transformation between the two poses of both systems can be compared directly.

We calculated the mean and standard deviation (std. dev.) of the absolute values for every axis of the translation and the rotation. The span of all movements was also recorded and is shown in Figure 1. To assess the influence of resolution and bayer color interpolation on the quality of the MBT, three different cameras were tested. The first one is a USB 3.1 $12 \mathrm{MP}$ global shutter color camera with a $12 \mathrm{~mm}$ lens. The second is the same camera as a black and white version. The third camera is a USB 3.0 $4 \mathrm{MP}$ global shutter color camera with a $6 \mathrm{~mm}$ lens.

\subsubsection{Results}

For the evaluation with the 12 MP color camera 149 measurements were conducted, for the $12 \mathrm{MP} \mathrm{B} / \mathrm{W}$ camera 77 measurements and for the $4 \mathrm{MP}$ color camera 41 measurements. The span of the specimen movements were in the range of up to $166 \mathrm{~mm}$ for the translation and up to 23 degrees for the rotation. We limited our tests to these small distances due to our industrial use case. This is explained in more detail down below. The distance from the specimen to the camera varied roughly between 1,5 to 3 meters. The coordinate system of the specimen sets the z-axis as up axis and uses a right handed coordinate system. Compared to the transformation between the two measured poses of the ground truth data, the MBT achieved results in the submillimeter range for all three camera types and for both camera resolutions. Rotation values are accurate to the second decimal place for nearly all values. It can be seen that with a higher resolution the results of the MBT improve in comparison to the ground truth data. These improvements apply to the mean and to the standard deviation of the absolute values of the difference between the two poses as well. To investigate the impact of Bayer demosaicing a measurement series with a B/W camera was conducted. Differences in the results are hardly noticeable and in most cases only concern the second decimal place. Considering the results in total we decided to use the $12 \mathrm{MP}$ color camera for our further experiments and the industrial use case. The improvements of the B/W camera in the results were small and the usage of color information gives us further opportunities in our industrial use case considering coated car bodies.

\subsection{Faro arm and Faro laser tracker with car body shell}

The second experiment was conducted with a car body shell on a locomotive platform. The platform could be moved along one axis in each of its directions. For ground truth measurements a Faro arm was used. Since the range of the Faro arm is limited we also used a Faro laser tracker for contactless measurements of larger platform displacements.

\subsubsection{Test setup}

The test setup is similar to the one before. In an initial position the body shell was measured by one of the Faro devices and then by the MBT system. Then the locomotive platform was moved along the $\mathrm{x}$-axis and the measurement of both systems was repeated. The transformation between the two measured poses for both systems were calculated and the mean and standard deviation of the absolute values of the transformation were determined per axis. To overcome the limitation of moving the body shell in the $\mathrm{x}$ axis only, we also moved the body shell in the $\mathrm{y}$ direction by hand. To consider movements on the $\mathrm{z}$ axis (up), we put objects under the body shell in order to lift it up. The span of all movements was recorded again. For the evaluation of the $12 \mathrm{MP}$ and the $4 \mathrm{MP}$ color camera we used the same setting as described above. The CAD model of the body shell was delivered by the planning department and can slightly differ to the real body shell up to a range of $\pm 0.5 \mathrm{~mm}$.

\subsubsection{Results}

For the evaluation with the 12 MP color camera, 139 measurements were conducted and for the $4 \mathrm{MP}$ color camera there were 87 measurements. The span of the movements of the specimen was in the range of up to $278 \mathrm{~mm}$ in the translation and up to 2 degrees in the rotation. The rotational movements were also adjusted by hand and due to the weight of the body shell, these 

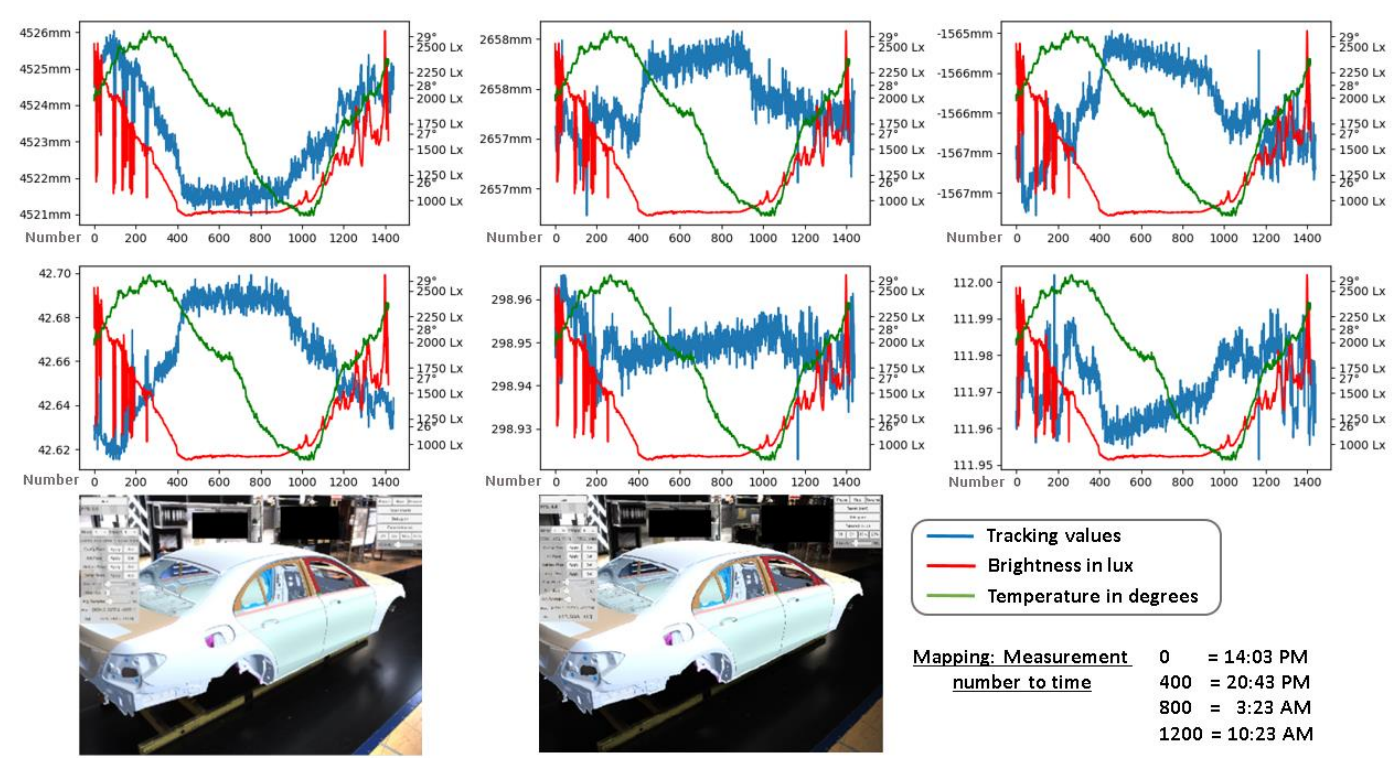

Figure 3: Lighting test results over 24 hours. Horizontal axis: measurement number. Vertical axis left: top row, translation in $X, Y$ and $Z$ in millimeters and mid row, rotation in $x, y$ and $z$ in degrees. Vertical axis right: Brightness in lux and temperature in degrees

rotational values are small. The right handed coordinate system of the body shell sets the z-axis as up axis. The $\mathrm{x}$ axis points along the car main axis and the $y$ axis points through the mid of the front wheel on the right. Compared to the ground truth data for the translation, the results of the MBT lie in the submillimeter range and are accurate to the second decimal place for the rotation. This applies to both cameras and resolutions as shown in Figure 2. The usage of the $4 \mathrm{MP}$ camera seems to have a slight advantage considering the accuracy but both results are close together. The total amount of measurements captured with the 4 MP camera is smaller and may look different if the same amount of measurements would have been conducted. Nevertheless we can assess a high accuracy for the MBT compared to the ground truth data for the considered span of movements in our tests. Since the results of the $12 \mathrm{MP}$ camera were slightly better than the results of the 4 MP camera in the tests with the test specimen, we decided to use the $12 \mathrm{MP}$ for our further experiments.

\subsection{Lighting and temperature over time}

The third experiment investigates the influence of lighting and temperature changes over time to the MBT. The tests were conducted under real operational conditions of our industrial use case. We set a body shell on a conveyor in a production hall and triggered a measurement with the MBT system every minute for 24 hours. We also measured the brightness of the scene in lux and the temperature in degrees Celsius. Figure 3 shows the results and correlations of the particular data for a 24 hour test with 1440 measurements. Besides, the outside lighting conditions at the test location had a direct effect to the indoor lighting due to sloped roof windows with milk glass. This caused some abrupt peaks in the results that can be seen in Figure 3. We started our tests at 2 p.m.. The mapping of measurement numbers to time stamps is depicted in Figure 3 for chosen values. As expected, the lighting conditions correlate directly to the results of the MBT. This can be seen best for the translation in $\mathrm{x}$ direction in Figure 3. Comparing the temperature and tracking results, a correlation is not as obvious. In Figure 3 the translation in $\mathrm{z}$ may show a trend that with sinking temperature the $\mathrm{z}$ values also decrease. The same is observed for the rotation around the $\mathrm{z}$ axis.

\begin{tabular}{|c|l|l|l|l|l|l|}
\hline & $\mathbf{X}$ & $\mathbf{Y}$ & $\mathbf{Z}$ & $\mathbf{R o t X}$ & RotY & RotZ \\
\hline Span & 3.28 & 1.66 & 1.68 & 0.04 & 0.03 & 0.03 \\
\hline $\boldsymbol{\mu}$ & 4508.6 & 2668.8 & -1571.2 & 42.80 & 299.14 & 112.23 \\
\hline $\boldsymbol{\sigma}$ & 0.35 & 0.35 & 0.28 & 0.006 & 0.006 & 0.007 \\
\hline
\end{tabular}

Table 1. Results of the 24 hour test with span, mean $(\mu)$ and standard deviation $(\sigma)$ values.

Translations are in $\mathbf{m m}$ and rotations in degrees.

We tried different camera settings and achieved the best and most stable results considering the span and standard deviation of the results with an automatic exposure. The results of a 24 hour test is shown in Table 1 . The MBT lies in the $2 \sigma$ range of $\pm 0.7 \mathrm{~mm}$ for the translation and $\pm 0.014^{\circ}$ for the rotation. Regarding the total span, we found a total range of up to $3.28 \mathrm{~mm}$ for the translation that is caused by some outliers.

\subsection{Noise}

The fourth experiment aims towards the investigation of the jitter/noise behavior of the MBT. The setting for this test was the same given in the third experiment where a body shell in a production hall was used. Therefore 30 measurements of the MBT were taken successively. Table 2 shows results for one of these tests with 30 measurements. We repeated these tests 


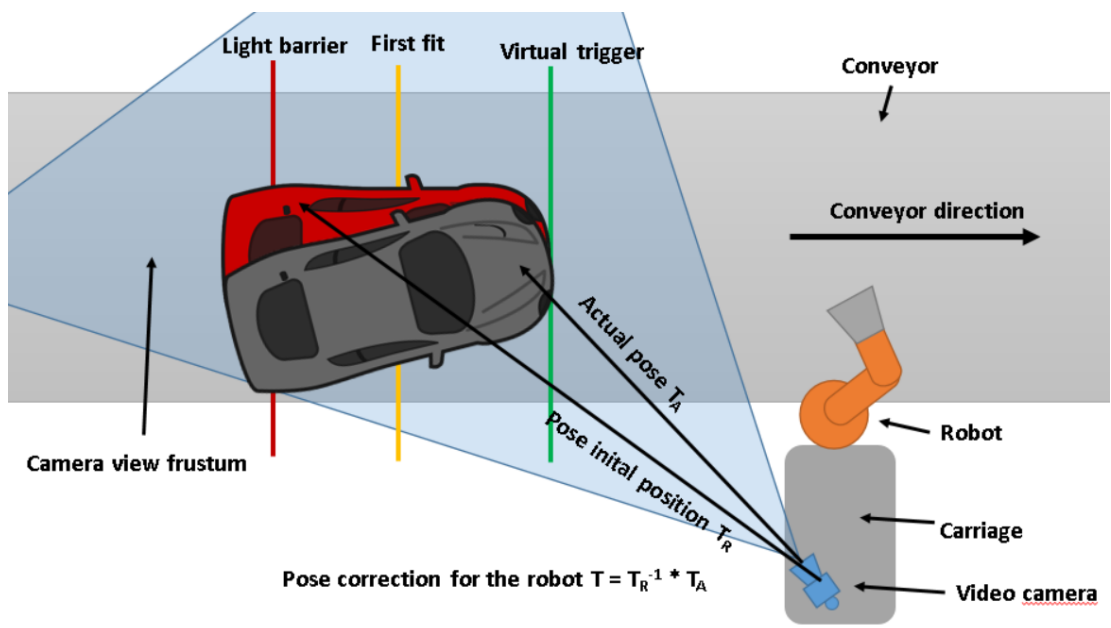

Figure 4: System setup for the robot measurement evaluation.

various times throughout our evaluation. It can be stated that the std. dev. throughout all tests only varied in the second decimal place for the translation and in the third decimal place for all rotation values. Table 2 also shows the column "dist". It contains the maximum distance to the mean value and is helpful to get an overview of the peaks of the values. Considering the fact that the values are given in $\mathrm{mm}$ and degrees, we found the MBT to be very stable and it achieves good results concerning jitter and noise.

\begin{tabular}{|c|l|l|l|l|l|l|}
\hline & $\mathbf{X}$ & $\mathbf{Y}$ & $\mathbf{Z}$ & RotX & RotY & RotZ \\
\hline dist & 0.12 & 0.16 & 0.21 & 0.003 & 0.003 & 0.005 \\
\hline $\boldsymbol{\mu}$ & 4521.21 & 2659.38 & -1565.02 & 42.67 & 298.93 & 111.99 \\
\hline $\boldsymbol{\sigma}$ & 0.04 & 0.07 & 0.08 & 0.001 & 0.001 & 0.001 \\
\hline
\end{tabular}

Table 2: Results of the noise test with maximum distance to the mean, mean $(\mu)$ and standard deviation $(\sigma)$ values.

\subsection{Setup and dismantling of the camera}

The fifth experiment deals with the question if a camera can be physically dismantled and set up again while achieving similar tracking results afterwards. Therefore a special camera mount was needed. We

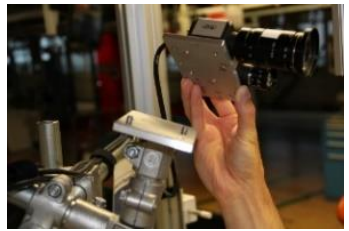

Figure 5: Camera dismantling with pinned fitting.

mounted the camera on a steel plate that was mounted on another steel plate with a connection of a pinned fitting (Figure 5). For the tests we measured a body shell with the MBT. Then we removed the alignment pins and the camera and then put the camera and the pins back on the second plate. Afterwards, a MBT measurement was triggered. We repeated this procedure six times.

Table 3 shows the mean and the std. dev. values of the absolute difference of the values per axis. The worst result is a submillimeter mean value of $0.8 \mathrm{~mm}$ on the $\mathrm{z}$ axis. The rotation only varies in the second decimal place. For applications requiring a dismantling of cameras in a calibrated setting this may be of interest to assess the impact on the accuracy.

\begin{tabular}{|c|c|c|c|c|c|c|}
\hline & $\mathbf{X}$ & $\mathbf{Y}$ & $\mathbf{Z}$ & RotX & RotY & RotZ \\
\hline $\boldsymbol{\mu}$ & 0.086 & -0.046 & 0.822 & -0.010 & 0.067 & 0.049 \\
\hline $\boldsymbol{\sigma}$ & 0.208 & 0.244 & 0.166 & 0.004 & 0.014 & 0.014 \\
\hline
\end{tabular}

Table 3: Results of camera setup and dismantling with mean $(\mu)$ and standard deviation $(\sigma)$ values.

\section{APPLICATION AND EVALUATION IN A PRODUCTION LINE}

After the general investigations concerning the accuracy and stability of the MBT we show the application and evaluation in an industrial scenario. Therefore the MBT is used to track body shells on a conveyor. The results are send to a robot in order to navigate to points where gaps should be measured. Collisions with the body shell should be avoided.

\subsection{Motivation of the scenario}

Most industrial robot applications rely on an installation and configuration step in which all components are put into operation. For our use case the points for the gap measurements are taught to a robot on a body shell in an initial position. Thereby the conveyor stands still. The body shells are mounted on skids. During system operation the conveyor is moving and skids with the body shells are automatically placed on the conveyor by machines. Afterwards the position of the skids can deviate from the initial position. To avoid these deviations that can cause a collision between a robot and the body shells, the deviations have to be measured to correct the robots movements. To provide accuracy, the conveyor, the systems engineering and the imaging device for the MBT have to be synchronized.

\subsection{System setup and general procedure}

Figure 6 shows the setup of our industrial use case. 


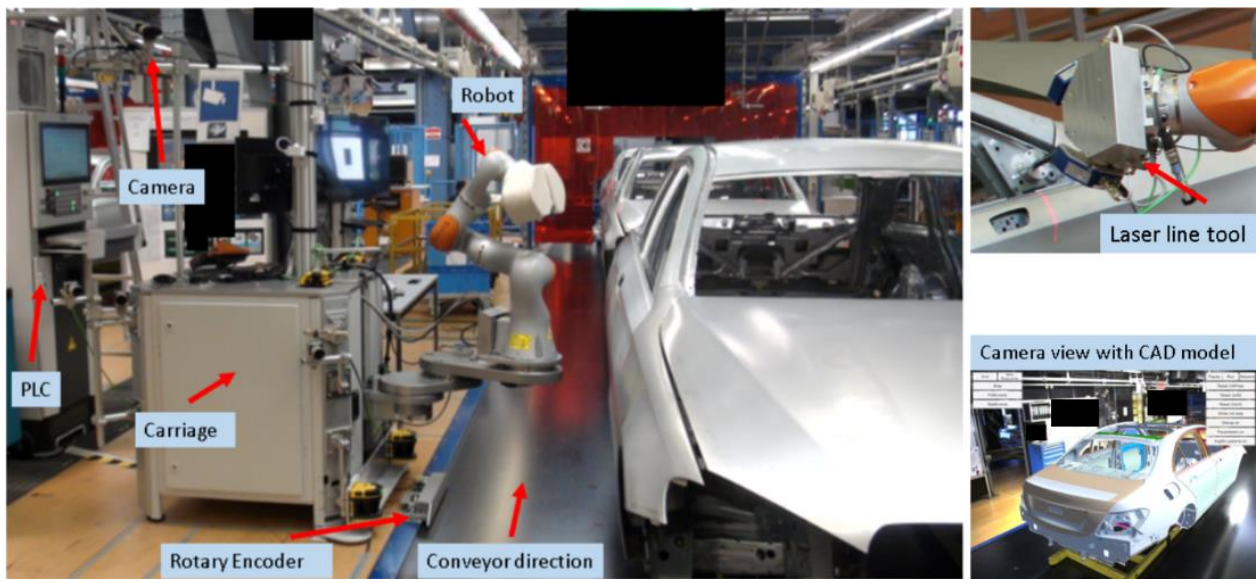

Figure 6: Real setup for the robot gap measurement evaluation. Critical information is blackened.

The MBT runs on an industrial PC in a carriage. In addition a light weight robot is attached to the carriage. The carriage and the robot are connected with a programmable logic controller (PLC) that controls the production station. When a body shell enters the station boundary, our system gets pieces of information about the car type and loads the corresponding CAD model in the MBT system. In the setup phase an initial position of the car is defined and the robot is calibrated for that position. The pose of the body shell is measured with the MBT and stored as reference pose $T_{R}$. Later on, this position on the conveyor is used as measurement position for the MBT and denoted as "virtual trigger". From this virtual trigger we calculate an earlier position in the opposite conveyor direction. This position is denoted as "first fit" (see Figure 4) and marks the position were the MBT matches the edges of the CAD model and video data to obtain a continuous tracking for the following frames. To account for the conveyor position and possible conveyor backlashes the increment of the conveyor is stored as $\operatorname{Inc} c_{R}$. Within the production process, cars come along the conveyor and are shifted or twisted in comparison to the reference pose. At the virtual trigger the actual pose $T_{A}$ is measured with the MBT and the transformation between $T_{R}$ and $T_{A}$ is calculated with $T=T_{R}^{-1} * T_{A}$.

\subsection{Synchronization between conveyor and model-based tracking}

To synchronize the MBT with the conveyor and the robot we present a method that does not require external time severs. The conveyor increment is determined by a separate rotary encoder that uses a gear to attach to the conveyor. With a conversion factor the covered distance of the conveyor can be calculated. When a car enters the station, a light barrier detects it (see Figure 4) and sets a conveyor increment counter to zero. After this, the PLC sends the actual conveyor increment $I n c_{A}$ every 3 milliseconds (ms) to our system. If $I n c_{A}$ is greater than $I n c_{R}$ we know that the virtual trigger position is reached and a timestamp $t_{a c t}$ is set in our system. Then the last pose $P_{t 1}$ before the timestamp and the first pose $P_{t 2}$ after the timestamp are used in combination with the time difference $t_{d i f f}$ to estimate the interpolated pose $P_{V T}$ at the virtual trigger. With the help of the relation $P_{V T} / t_{\text {diff }}=$ factor we can use the slerp function to interpolate between the poses $P_{t 1}$ and $P_{t 2}$. Doing so, the correct pose $T_{A}$ at time $t_{\text {act }}$ for the virtual trigger position can be calculated. $T_{A}$ is directly comparable to the pose $T_{R}$ of the reference position. Since the PLC sends the increments every $3 \mathrm{~ms}$ to the MBT system an additional maximum error of $0.225 \mathrm{~mm}$ can occur. The conveyor speed was $75 \mathrm{~mm}$ per second in our experiments.

\subsection{Error sources}

In this complex application scenario various error sources influence the accuracy. Below the most important ones under an assumption of $2 \sigma$ are listed:

- MBT errors: $0.7 \mathrm{~mm} \pm 1.5 \mathrm{~mm}$

- MBT-conveyor synchronization:1.7 $\pm 1.5 \mathrm{~mm}$

- Robot to conveyor synchronization: $\pm 0.5 \mathrm{~mm}$

- Robot calibration of gap points: $\pm 0.5 \mathrm{~mm}$

- Production tolerance of test object $\pm 0.5 \mathrm{~mm}$

- Calibration conveyor direction

- Calibration robot to test object

\subsection{Evaluation of the synchronization}

To test our synchronization method we put a coated car on a conveyor that can be moved forward and backward. The reference pose was measured in an initial position with the MBT system and the conveyor increments were recorded. Then the conveyor with the car was moved in the opposite conveyor direction in a start position before the virtual trigger

For the tests the conveyor was started and the measurements with the MBT were triggered by reaching the increment values of the virtual trigger. For every measured pose the transformation to the reference pose was calculated. We repeated this procedure 56 times. Ideally, the mean of the pose differences should be zero if no error would occur. The mean, standard deviation and span of the results is 
depicted in Table 4. In this test the MBT errors and the production tolerances have an influence on the results. As expected, the biggest error occurs on the $\mathrm{x}$-axis that corresponds to the conveyor direction. We found that coated cars are a bigger challenge for the MBT than body shells. This is caused by light reflections in the coating that can lead to edges being detected falsely and thus can corrupt the matching process. Unfortunately we have no comparison to ground truth data for coated cars. Therefore we rely on the assessment of the total error including all error sources described above. The rotational values only vary in the second decimal place. The translation values only vary in the submillimeter space except the $\mathrm{x}$-axis.

\begin{tabular}{|c|l|l|l|l|l|l|}
\hline & $\mathbf{X}$ & $\mathbf{Y}$ & $\mathbf{Z}$ & RotX & RotY & RotZ \\
\hline $\boldsymbol{\mu}$ & -1.72 & -0.19 & 0.08 & 0.00 & -0.02 & 0.03 \\
\hline $\boldsymbol{\sigma}$ & 0.78 & 0.24 & 0.20 & 0.01 & 0.01 & 0.01 \\
\hline span & 3.11 & 1.17 & 0.83 & 0.04 & 0.05 & 0.04 \\
\hline
\end{tabular}

Table 4: Evaluation results of the synchronization method with mean $(\mu)$, std. dev. $(\sigma)$ and span.

To be able to assess and quantify the total error of our system with a more independent measurement method a further evaluation is presented in the next section.

\section{Robot measurements}

In section 4.4 the error sources of the complete system are described. In a complex setup described so far, various correlations between the error sources exist. Thus a distinguished assessment of the influence of a single error source is hard to determine. We tried to quantify single error sources in this paper to allow an easier assessment of single components and the behavior of the MBT. Finally, the total error is essential to assess the accuracy of the whole system. Therefore we present a method to measure the total error. This is done by using the light weight robot of the gap measurement use case. On the robots end effector a tool for gap measurements is attached, denoted as laser line tool (LLT). It consists of two laser line scanners that are arranged in a mount in the way that a large laser scan line is generated. The LLT is shown in Figure 6, once with and once without housing. If the robot approaches a measurement point at a car the deviation of a gap mid point to the LLT mid point can be measured. This gives us the total error in 2D LLT coordinates. With this criterion it can at least be stated how precise a measurement point of an actual car can be measured with the robot LLT and gives us an overview of how the synchronized MBT is suited for this kind of application.

As described in section 4.1 all necessary measurement points on the body shell are taught to the robot in an initial position. The LLT is thereby aligned perpendicularly to the normal of a gap, e.g. the gap between the hood and the car wing (see Figure 8). To assure a precise alignment a template for the alignment of the laser line is used (see Figure 8).
The template has little bridges on the backside that fit into a gap and ensure a tight application of the template. The laser line is then aligned orthogonally to the template by hand and the robots position is saved. Afterwards a software offset is calculated on the basis of the measurements that corrects small deviations and ensures a tight orthogonal alignment of the laser line to the gap.

During the system setup in the initial position the conveyor direction and the transformation between the robot base and the body shell is measured with a high precision measurement device, e.g. a Faro laser tracker. In the conveyor synchronous mode the robot uses these values to predict the position of the body shell at various conveyor increments. Together with the pose correction values of the MBT and the calibrated measurement points this makes an accurate approach of the robot to each measurement point possible. For the evaluation we used three measurement points on the body shells. Looking at the side of a car, the first is a horizontal gap between the trunk lid and the car body. On this gap our measurement point P1 is approximately placed in the mid. The second gap is vertical and between the back driver door and the car body. The measurement point $\mathrm{P} 2$ lies on the left side of the right wheel housing at the lower end. The third gap is also vertical and between the driver door and the back driver door. The measurement point $\mathrm{P} 3$ is placed in the upper area.
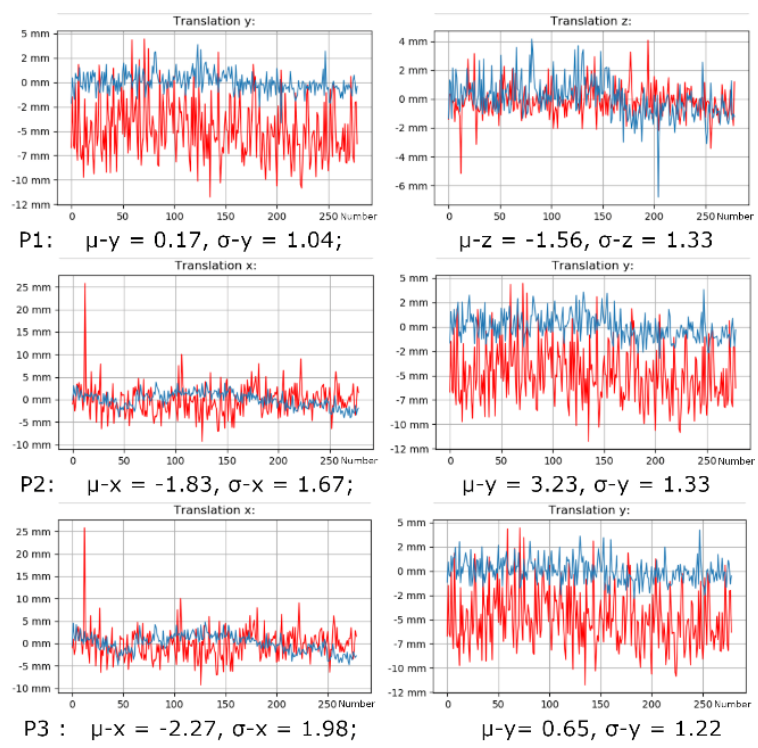

Figure 7: Results of robot measurements for three gap points. MBT results are marked in red for the respective axis and robot results in blue.

In our evaluation the MBT measures the body shell at the virtual trigger and sends the pose correction to the robot. The robot uses the values and his prediction of the body shell to correct the calibrated measurement positions in his coordinate system. Then the robot measures three points successively as the body shell 
on the conveyor passes. The robot approaches each gap in a way that the laser line is orthogonal to the gap. In our experiment, three gap points were measured 278 times. The results are shown in Figure 7. For each measurement point the axis is denoted in analogy to the car coordinate system (see Figure 7). The origin of the car coordinate system is in the mid of the front suspension. The $\mathrm{x}$-axis is along the cars main axis and directed to the back wheels, the y-axis is directed to the right front wheel and $\mathrm{z}$ is the up-axis.

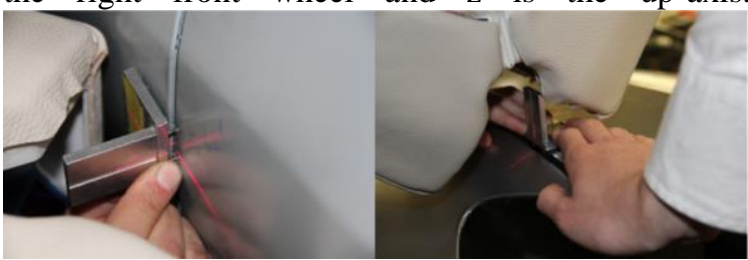

Figure 8 Laser line tool calibration to gaps.

The robot started each of the 278 measurement series with $\mathrm{P} 1$ then measured $\mathrm{P} 2$ and $\mathrm{P} 3$. As shown in Figure 6 the cars back enters the station first. The body shells length is approximately 4.5 meters. For an ideal situation, the blue robot measurements in Figure 7 would be nearly zero, indicating that the MBT results in red correct the robot movements perfectly. It can be seen in Figure 7 that the mean for the vertical gaps increase over the length of the body shells. Regarding the std. dev., a slight increase can be observed too. The further away the measurement points are in X-direction the more uncertainty can be found in the results. This is mainly caused by the leverage of rotational errors of the MBT, of the conveyor direction calibration and of the transformation between the body shell and the robot. Under the assumption of a gauss distribution of $2 \sigma$, it can be stated that P1 can be measured with an accuracy of up to $4.22 \mathrm{~mm}$, given the worst case. P2 with an accuracy of up to $5.89 \mathrm{~mm}$ and P3 with an accuracy of up to $6.23 \mathrm{~mm}$ in the worst case. The laser line has a length of several centimeters (see Figure 8). Thus, gaps can be measured with the presented method. To further improve our method, we plan to introduce a second virtual trigger that is shifted about a half or one-third of a body shell after the virtual trigger. This will reduce deviations caused by leverage effects of rotational errors or rising uncertainties of the matching pose by the MBT over larger distances.

\section{CONCLUSION}

In this paper we presented several methods to evaluate the accuracy and stability of model-based tracking approaches. We showed tests to determine the accuracy with different camera resolutions or camera types, the influence of light and temperature changes and the robustness to noise. For one particular approach we found that with precise CAD models an accuracy in the submillimeter range for the translation and up to the second decimal place for the rotation can be achieved. Distances of up to $300 \mathrm{~mm}$ and rotations of up to $23^{\circ}$ were evaluated. Furthermore a method was presented to synchronize the vision based tracking to a conveyor and robot. This synchronization was also evaluated. Finally, we presented the application of model-based tracking in an industrial gap measurement use case with a light weight robot. This complex system was evaluated and the total error determined. In future we plan to introduce a second virtual trigger to further improve the accuracy of the system and to evaluate the system for coated cars. The presented methods help to assess the applicability of model based tracking approaches for even complex industrial application scenarios.

\section{REFERENCES}

[Wue07] Wuest H., Wientapper F., Stricker D. (2007) Adaptable Model-Based Tracking Using Analysisby-Synthesis Techniques. In: Kropatsch W.G., Kampel M., Hanbury A. (eds) Computer Analysis of Images and Patterns. CAIP 2007.

[Com06] Comport, A., Marchand, E., Pressigout, M., Chaumette, F.: Real-time markerless tracking for augmented reality: the virtual visual servoing framework. IEEE Trans. on Visualization and Computer Graphics 12(4), 615-628 (2006)

[Vac04] Vacchetti, L., Lepetit, V., Fua, P.: Combining edge and texture information for realtime accurate 3d camera tracking. In: Proceedings of International Symposium on Mixed and Augmented Reality (ISMAR) (2004)

[Hin11] Hinterstoisser, S., Holzer, S., Cagniart, C., Ilic, S., Konolige, K., Navab, N.,Lepetit, V.: Multimodal Templates for Real-Time Detection of Texture-less Objects in Heavily Cluttered Scenes. IEEE International Conference on Computer Vision (ICCV), 2011

[Nie03] Nienhaus, M., Doellner, J.: Edgeenhancement - an algorithm for real-time nonphotorealistic rendering. In: WSCG (2003)

[Gar18] Garon,M., Laurendeau, D., Lalonde, J-F.: A Framework for Evaluating 6-DOF Object Trackers. In: Computer Vision - ECCV 2018.

[Wu17] Wu, P-C., Lee, Y-Y., Tseng, H-Y., Ho, H-I., Yang, M-H., Chien, S-Y: [Poster] A Benchmark Dataset for 6DoF Object Pose Tracking. ISMARAdjunct, 2017.

[Hod17] Hodaň,T., Haluza, P., Obdržálek, Š., Matas, J., Lourakis, M., Zabulis, X.: T-LESS An RGB-D Dataset for 6D Pose Estimation of Texture-less Objects, IEEE Winter Conference on Applications of Computer Vision (WACV), 2017

[Far20] FARO Europe GmbH \& Co. KG, Faro arm, Faro laser tracker: https://www.faro.com, 2020

[Gom20] GOM GmbH, ATOS, https://www.gom.com, 2020. 